Revue internationale P.M.E.

Économie et gestion de la petite et moyenne entreprise

\title{
La création d'entreprise technologique comme une succession de projets
}

\section{Michel Bernasconi}

Volume 21, numéro 1, 2008

URI : https://id.erudit.org/iderudit/038005ar

DOI : https://doi.org/10.7202/038005ar

Aller au sommaire du numéro

\section{Éditeur(s)}

Presses de l'Université du Québec

ISSN

0776-5436 (imprimé)

1918-9699 (numérique)

Découvrir la revue

Citer cet article

Bernasconi, M. (2008). La création d'entreprise technologique comme une succession de projets. Revue internationale P.M.E., 21(1), 119-142.

https://doi.org/10.7202/038005ar
Résumé de l'article

Il est aujourd'hui largement reconnu que le processus de création d'une entreprise technologique n'est pas linéaire, mais qu'il est fait de cheminements et que les écarts entre les prévisions et les réalisations sont la règle plutôt que l'exception. Pourtant, malgré des avancées, la littérature ne semble pas avoir été aussi loin que nécessaire pour prendre en compte cet état de fait. Ce travail propose donc un modèle adapté à ces situations. Il considère le processus de création d'une entreprise technologique comme la réalisation successive de trois projets : le projet d'affaires, le projet d'entreprendre et le projet d'entreprise. Chaque projet est constitué d'un système, c'est-à-dire d'éléments en interaction que l'entrepreneur doit piloter. L'approche de l'objet de recherche est de type constructiviste. Le modèle a été bâti par une confrontation de la littérature avec le matériel d'une étude de cas d'une jeune entreprise dans l'édition de logiciel en création. L'ambition de ce travail est d'offrir une représentation de l'entreprise technologique en création qui permette aux entrepreneurs et aux acteurs de l'accompagnement de mieux communiquer sur le processus de création afin d'en améliorer la réalisation.
Ce document est protégé par la loi sur le droit d'auteur. L'utilisation des services d’Érudit (y compris la reproduction) est assujettie à sa politique d'utilisation que vous pouvez consulter en ligne.

https://apropos.erudit.org/fr/usagers/politique-dutilisation/ 


\title{
La création d'entreprise technologique comme une succession de projets
}

Michel BERNASCONI

CERAM Sophia-Antipolis

\author{
MOTS CLÉS
}

\section{Création d'entreprises de haute technologie - Projet d'entreprise Modélisation du processus entrepreneurial}

\begin{abstract}
L'AUTEUR
MICHEL BERNASCONI est professeur de management et d'entrepreneuriat au CERAM Business School, situé au cœur du technopole de Sophia-Antipolis, France. II travaille dans le champ de l'entrepreneuriat technologique depuis une quinzaine d'années. II dirige le centre «entrepreneuriat technologique » du CERAM qui mène des actions dans les domaines de l'incubation, des méthodes d'accompagnement des créations d'entreprises. Adresse: CERAM SophiaAntipolis, 60, rue Dostoïevski, B.P. 085, 06902 Sophia-Antipolis, France. Courriel: <michel. bernasconi@ceram.fr>.
\end{abstract}

\section{RÉSUMÉ}

Il est aujourd'hui largement reconnu que le processus de création d'une entreprise technologique n'est pas linéaire, mais qu'il est fait de cheminements et que les écarts entre les prévisions et les réalisations sont la règle plutôt que l'exception. Pourtant, malgré des avancées, la littérature ne semble pas avoir été aussi loin que nécessaire pour prendre en compte cet état de fait. Ce travail propose donc un modèle adapté à ces situations. II considère le processus de création d'une entreprise technologique comme la réalisation successive de trois projets : le projet d'affaires, le projet d'entreprendre et le projet d'entreprise. Chaque projet est constitué d'un système, c'est-à-dire d'éléments en interaction que l'entrepreneur doit piloter. L'approche de l'objet de recherche est de type constructiviste. Le modèle a été bâti par une confrontation de la littérature avec le matériel d'une étude de cas d'une jeune entreprise dans l'édition de logiciel en création. L'ambition de ce travail est d'offrir une représentation de l'entreprise technologique en création qui permette aux entrepreneurs et aux acteurs de l'accompagnement de mieux communiquer sur le processus de création afin d'en améliorer la réalisation. 


\begin{abstract}
Nowadays it is widely acknowledged that the creation process of a technological enterprise is not linear; but that it is made of evolutions and that the gap between anticipations and accomplishment is more a convention than an exception. Yet, in spite of some progress, the literature does not seem to have been as far as needed to take into account this reality. Therefore, this study put forward a pattern that fits these situations. It deems the creation process of a technological enterprise as the successive execution of three projects: the business project, the entrepreneurial project and the enterprise project. Each project comprises a system, i.e. interacting elements the entrepreneur needs to manage. The approach of the research object is of a constructivist type. The pattern is based on a confrontation between the literature and the case study material of a young enterprise in software editing, currently in the creation phase. The ambition of this study is to give a representation of the technological enterprise in the creation phase that allows the entrepreneurs and the accompanying actors to better communicate on the creation process, in order to improve its execution.
\end{abstract}

\title{
RESUMEN
}

Hoy en día es ampliamente reconocido que el proceso de creación de una empresa tecnológica no es lineal, sino que se lleva a cabo a través de diferentes vías y que las diferencias entre las previsiones y las realizaciones constituyen más la regla que la excepción. Sin embargo, a pesar de los avances, la literatura no parece haber profundizado lo suficiente para tener en cuenta este hecho. Este trabajo propone entonces un modelo adaptado a este tipo de situaciones. Este modelo considera el proceso de creación de una empresa tecnológica como la realización sucesiva de tres proyectos: el plan de negocios, el proyecto de emprender y el proyecto de la empresa. Cada proyecto consta de un sistema, es decir, de elementos en interacción, que el emprendedor debe manejar. El enfoque del objeto de la investigación es de tipo constructivista. El modelo ha sido construído a través de un enfrentamiento de la literatura con el material de un estudio de caso de una empresa joven en la edición de un software en creación. La ambición de este trabajo es ofrecer una representación de la empresa tecnológica en creación que permita a los empresarios y a los actores del acompañamiento de comunicar mejor acerca del proceso creativo, con el fin de mejorar la realización.

\section{ZUSAMMENFASSUNG}

Es ist heute weithin anerkannt, dass die Gründung von technologischen Unternehmen kein linearer Prozess, sondern das Wandern auf einem Kriechweg ist, auf dem Abweichungen zwischen der Planung und der Realisation eher die Regel als die Ausnahme sind. Trotzdem scheint es, als ob sich die Literatur auf diesem Gebiet noch nicht so weit entwickelt hat. Diese Arbeit schlägt ein Modell vor, welches diese Entwicklungen berücksichtigt. Die Realisierung der technologischen Unternehmensgründung wird als drei aufeinander folgende Projekte betrachtet: Das Geschäftsprojekt, das unternehmerische Projekt und das Unternehmensprojekt. Jedes dieser Projekte kann in dem Sinne als System angesehen werden, 
als der Unternehmer die Wechselwirkung seiner Aktionen steuern muss. Das Modell wurde aufgrund der Unterschiede in der Literatur und einer Fallstudie einer jungen Software-Entwicklungsfirma erstellt. Das Ziel der Arbeit besteht darin, ein HightechUnternehmen in der Gründungsphase vorzustellen, um Unternehmern und Personen die Unternehmen begleiten, den Prozess der Unternehmensgründung besser zu kommunizieren und schlussendlich die Realisation besser vollziehen zu können.

\section{Introduction}

La compréhension du processus de création des entreprises de haute technologie intéresse les chercheurs et les praticiens. Les uns et les autres reconnaissent qu'il est remarquable par sa complexité et son évolution.

Les chercheurs ont exploré à fond le processus entrepreneurial dont les approches ont été analysées et mises en perspective (Saporta, 1994; Hernandez, 2001; Verstraete, 2001; Fayolle, 2004). Pour prendre en compte plus particulièrement l'évolution, le processus entrepreneurial a été qualifié de chemin faisant, d'interactif avec des allers et retours (Marchesnay, 2002), ou avec des boucles rétroactives (Garnsey, 1996). Ces qualificatifs s'appliquent particulièrement à la jeune entreprise technologique qui se développe dans un contexte d'incertitude (Monsted, 2000). Toutefois, les différentes approches semblent insuffisantes pour restituer l'ampleur de l'évolution que connaissent les projets dans la haute technologie et il paraît intéressant de considérer que l'évolution et la recomposition du projet sont la règle plutôt que l'exception. D'ailleurs, dès 1984, Massacrier et Rigaud mettaient en évidence les écarts entre les prévisions et les réalisations et soulignaient la nécessité d'approches qui permettraient aux entrepreneurs de simuler leur projet. Ce besoin de simulation reste d'actualité, si l'on accepte l'idée que les modèles et approches proposés ne semblent pas adaptés pour éclairer les praticiens.

Les investisseurs et les acteurs de l'accompagnement reconnaissent également la complexité et l'évolution du processus de création de la jeune entreprise technologique. Ils savent par expérience que le projet va profondément évoluer et que le premier plan d'affaires n'a pratiquement aucune chance d'être réalisé en l'état. Les plus compétents induisent des modèles opératoires à partir de leurs pratiques. Ceux-ci restent très contingents et ne leur permettent pas de disposer d'un modèle d'ensemble, qu'ils pourraient partager avec les créateurs. Les entrepreneurs, hormis les récidivistes, abordent la création en agissant sur des éléments, découvrant chemin faisant les liens et les interactions. Ils n'ont pas non plus une représentation d'ensemble de l'aventure de la création d'entreprise dans laquelle ils se lancent. 
L'objectif de ce travail est donc de proposer une approche globale de la création de la jeune entreprise technologique qui rende mieux compte de ses évolutions et de son cheminement. Son ambition est de constituer une représentation commune aux entrepreneurs et aux acteurs de l'accompagnement, leur permettant ainsi d'anticiper et de suivre le développement des jeunes entreprises technologiques. Il s'agit en fait de définir un métamodèle qui pourra ensuite s'adapter à la particularité de chaque création d'entreprise.

Le travail s'appuie sur les théories existantes, pour les compléter dans une démarche de recherche constructiviste. Une représentation composite a été bâtie à partir des approches processuelle, systémique et projet. Elle considère les premières années de la jeune entreprise technologique comme une succession de trois projets, qui seront plus ou moins proches les uns des autres. Chacun des projets étant par ailleurs défini comme un système ouvert dont les entrepreneurs doivent assurer le pilotage.

Amener les entrepreneurs à envisager que leur enjeu est de réussir successivement trois projets brise la représentation mentale d'une évolution basée sur le passage plus ou moins linéaire d'un état à un autre. La dimension projective du modèle facilite l'acceptation de reformulation du projet comme une situation naturelle et les amène à accepter l'idée que la réussite de leur projet réside plus dans leur capacité à gérer l'évolution que dans la pertinence de la stratégie initiale décrite dans le plan d'affaires.

Dans une première partie, la littérature sera rapidement parcourue pour en extraire les éléments constitutifs du modèle proposé. Dans une deuxième partie, la méthodologie de l'étude du cas unique sera justifiée et celui-ci décrit. Enfin, la troisième partie proposera un modèle en s'appuyant sur l'étude de cas.

\section{Les enseignements des travaux sur la création d'entreprises technologiques}

Les jeunes entreprises technologiques ne sont pas nécessairement aisées à définir si l'on se réfère aux nombreuses appellations de la littérature: firmes de hautes technologies, entreprises de technologies avancées, entreprises innovantes, mais aussi «new technology based firms» ou encore «knowledge based-firms». De nombreux critères ont été proposés pour qualifier l'entreprise technologique (Cooper, 1986; Albert et Mougenot, 1998). Baruch (1997) propose de retenir trois critères principaux pour différencier une entreprise de haute technologie des autres entreprises:1) le niveau d'éducation du personnel, 2) l'investissement en R-D, 3) le secteur industriel de l'entreprise. 
De plus, les jeunes entreprises technologiques innovantes ont quelques caractéristiques singulières. Elles sont situées dans des secteurs naissants et instables dont elles tirent parti. Elles ont des relations étroites avec les milieux scientifiques dont les entrepreneurs sont fréquemment issus. Elles ont des difficultés à identifier et capter les premiers marchés. Les besoins en investissement sont élevés, en augmentation au cours du développement et sont assumés par des investisseurs spécialisés. Pour toutes ces raisons, les auteurs insistent sur l'incertitude et le risque qui caractérisent ces entreprises (Monsted, 2000). Avec toutes ces incertitudes, il existe le plus souvent un écart entre les prévisions et les réalisations (Massacrier et Rigaud, 1984), en particulier dans l'offre et dans la mise en œuvre (Bernasconi et Moreau, 2003).

Au-delà de ces critères, la dimension temporelle pose également des difficultés pour définir l'entreprise de haute technologie. La période au cours de laquelle on considère qu'une entreprise est en période de création, ou de démarrage, mérite à ce titre également d'être précisée. On peut considérer avec Tesfaye (1997) que la période de création s'achève avec la vente du premier produit. On peut également prendre l'atteinte du seuil de rentabilité comme l'étape du passage de la création au développement. Les observations de Twaalfhoven et Kugi (2003) sur 200 entreprises technologiques montrent que le seuil de rentabilité est atteint en moyenne au bout de 60 mois, alors que les plans d'affaires faisaient apparaître un délai moyen de 30 mois, soit un rapport de 1 à 2 . Sammut (2001), dont le champ d'observation n'était pas la technologie, considère que la phase de démarrage se termine lorsque le dirigeant fait preuve d'une conscience stratégique, c'est-à-dire adopte une gestion systémique, engage un transfert organisationnel et est en mesure d'atteindre ou de dépasser le seuil de rentabilité. Cette approche a sans doute pour effet d'allonger le nombre d'années nécessaire. En retenant l'atteinte du seuil de rentabilité, nous pouvons donc considérer que la période de création d'une entreprise technologique se situe entre trois à sept ans.

Les jeunes entreprises de haute technologie en création ayant été définies, la partie suivante se propose de parcourir les différentes approches de l'entrepreneuriat en mettant en évidence leur pertinence pour ce type de création d'entreprises.

\subsection{Les approches traditionnelles}

Inspiré par l'analogie avec la biologie, un fort courant de recherche historique s'est intéressé à identifier les phases par lesquelles passe une entreprise à partir de la création. Les premiers travaux de Greiner (1972), de Quinn et Cameron (1983) et de Churchill et Lewis (1983) ont identifié des étapes séquentielles 
auxquelles on peut associer des logiques d'organisation. Des critiques ont été faites, remettant en cause le déterminisme et la séquentialité des phases (Eggers, Leahy et Churchill,1994;Albert, Fayolle et Marion, 1994; Churchill, 1998) ainsi que la sous-conceptualisation du développement (Garnsey, 1996). Dans la haute technologie, des modèles de cycle de vie concentrés sur les phases initiales ont été déterminés (Gordon-Bell et McNamara,1991; Tesfaye, 1997 ; Klofsten, 1997). À titre d'exemple, celui de Gordon-Bell et McNamara (1991) propose des phases (concept, faisabilité, développement du produit, développement du marché, croissance) couplées avec une évolution des compétences de l'organisation. Ces modèles plus spécifiques demeurent encore trop séquentiels et simplificateurs et ne permettent pas de rendre compte de la redéfinition de l'activité et de la reconfiguration des ressources au cours de la vie de l'entreprise.

L'approche processuelle qui s'est beaucoup développée au cours des 20 dernières années tente de répondre à la question suivante: comment naissent les organisations? Pour Gartner (1985), un entrepreneur est celui qui crée une organisation et la focalisation se fait donc sur l'émergence organisationnelle. Pour décrire le processus de la création d'une nouvelle activité, Gartner a proposé un modèle interactionniste qui comporte quatre dimensions (environnement, individu, processus et organisation). Pour Bygrave et Hofer (1991), le processus entrepreneurial implique toutes les fonctions, activités et actions orientées vers la perception d'opportunités et la création d'organisations pour les atteindre. Ils proposent d'ailleurs de décrire ce processus entrepreneurial comme la succession de trois phases: la reconnaissance d'une opportunité, l'exploitation de l'opportunité et la création de valeur. De nombreuses propositions complémentaires ont été faites ultérieurement sur la même logique.

L'approche processuelle identifie les fonctions ou dimensions du modèle et décrit les interactions à différents moments de la vie de l'entreprise. Toutefois, elle ne met pas suffisamment l'accent sur les retours en arrière et les redéfinitions du projet, c'est-à-dire les discontinuités.

L'approche basée sur la notion d'opportunité entrepreneuriale de Venkataraman (1997), et Shane et Venkataraman (2000) met l'accent sur la manière dont les opportunités de créer de nouveaux produits ou services sont découvertes et exploitées. Ici, c'est donc moins l'émergence de l'organisation qui est privilégiée que l'émergence de l'activité. Toutefois, cette approche ne prend pas en compte le long processus de transformation de l'opportunité en activité, avec les évolutions de l'offre, en particulier celles relatives au couple produit-marché. 
De ces nombreuses approches, nous retiendrons plus particulièrement, pour le modèle proposé, que le développement de la nouvelle entreprise se fait en trois phases, comme celles définies par Bygrave et Hofer: reconnaissance d'une opportunité, exploitation de l'opportunité et création de valeur.

\subsection{L'analyse systémique}

Le paradigme systémique (Le Moigne, 1977) considère qu'il est inutile d'examiner une organisation comme une unité discrète, isolée des forces sociales, de la culture et des institutions qui facilitent et affectent son activité économique. Dans l'approche systémique, l'attention se porte sur la gestion des interactions entre les éléments constitutifs du système. Dès lors, elle permet d'appréhender l'évolution de l'entreprise plutôt comme un processus de mise en œuvre que comme un processus de décision, mettant en évidence le caractère émergent de la stratégie. L'approche systémique est aussi par essence téléologique et, par conséquent, elle induit une représentation de la création d'entreprise comme une succession de réorganisation de la relation entre la fin et les moyens, c'est-à-dire entre les objectifs et la mise en œuvre.

Les auteurs, qui ont défini la création d'entreprise comme un système, ont identifié les éléments constitutifs du système, encore appelés dimensions ou attributs. Bruyat (1994) rappelle le consensus de la communauté scientifique sur quatre dimensions nécessaires pour l'étude de la création d'entreprise: le créateur, le processus de création, l'environnement et l'entreprise nouvelle. Sammut (2001) propose d'observer le processus de création en prenant en compte simultanément cinq variables clés qui sont:l'entrepreneur, les ressources financières, l'environnement, l'organisation et l'activité. Nous avons retenu ces cinq variables, que nous appellerons éléments du système. Ils vont être présentés successivement en mettant en évidence leurs singularités dans la création des jeunes entreprises technologiques.

\subsection{L'entreprise comme un projet}

L'entreprise peut être observée comme un projet. Cet usage n'est pas naturel, car un projet est traditionnellement défini comme une unité organisationnelle vouée à l'atteinte d'un but, dans le cadre d'un budget et en conformité avec des spécifications de performance prédéterminées (Gaddis, 1959). Toutefois, certains auteurs considèrent que la firme basée sur le projet (project-based firm) est une nouvelle manière de penser les organisations et leur management (De Fillippi et Arthur, 1998) ou encore que la théorie 
générale du management de projet peut être appliquée à tout projet, y compris une organisation (Söderlund, 2004). Knights et Mueller (2004) proposent de considérer la stratégie comme un projet sans fin qui permet de prendre en compte simultanément la formulation et la mise en œuvre, dans un processus continuel d'autoformation et de reconstruction. L'approche de Bréchet (1994) relative à la transformation du projet d'entreprendre en projet d'entreprise est particulièrement intéressante dans le domaine de la création et du développement des jeunes entreprises. Bréchet considère qu'au cours de cette transformation ce n'est pas l'organisation qui est permanente mais les attributs du projet, eux-mêmes évoluant au cours de la vie du projet. Ces attributs évolutifs sont: les objectifs du porteur de projet, le triplet technologie-produit-marché et la mise en œuvre.

Dans le cas du processus de création d'entreprises technologiques, nous retiendrons de l'approche projet qu'elle réconcilie la formulation et la mise en œuvre. À Bréchet nous emprunterons, en l'adaptant, la transformation du projet d'entreprendre en projet d'entreprise, ainsi que les attributs évolutifs du projet.

Les trois grands courants de travaux pris en compte apportent chacun des éléments pertinents pour la tentative de modélisation. Les approches traditionnelles ont identifié des phases différentes de la vie de la jeune entreprise dont le modèle s'inspirera. De l'approche systémique, nous retiendrons les cinq éléments et leurs interactions dynamiques. Enfin, l'approche projet vient enrichir l'approche systémique avec les attributs évolutifs et le passage d'un projet à un autre.

Après avoir présenté les principales approches de la création d'entreprise dans la littérature, relevé les limites en ce qui concerne les jeunes entreprises technologiques et indiqué les éléments qui seront repris dans le modèle proposé, nous allons maintenant présenter la méthodologie de recherche.

\section{Enrichissement des travaux existants : une modélisation par le cas Mockup ${ }^{1}$}

L'objet de ce travail est de proposer un modèle de représentation du processus de création d'entreprise qui permette de mieux prendre en compte l'évolution que les modèles existants. Ce qui est nouveau, c'est tout d'abord une utilisation différente de concepts, comme le projet et les phases de développement.

1. Le nom de l'entreprise a été masqué. 
C'est également une prise en compte enrichie du système entrepreneurial. Le modèle a été élaboré par une confrontation de la littérature avec un cas, dans une démarche inductive qui s'inscrit dans une approche constructiviste.

\subsection{Méthodologie et terrain}

Lorsqu'on souhaite modéliser la création d'entreprise, il est nécessaire de disposer d'information sur la durée du processus. De ce fait, un cas longitudinal apparaît comme adapté. Dans cette partie, ce choix méthodologique va être justifié, puis le cas Mockup sera présenté.

\subsubsection{Choix méthodologiques}

La méthodologie s'appuie sur une étude cas unique. L'utilisation d'un seul cas est l'objet de controverse. Toutefois, Yin (1989) estime que le cas unique peut être assimilé à une expérimentation et qu'il peut se justifier dans certaines situations. Stake (1994) considère que le cas unique permet la mise au jour de phénomène d'un processus dont la compréhension enrichit la théorie. Il recommande de choisir les cas susceptibles d'enseignement les plus riches, qui ont le plus fort potentiel d'apprentissage. La validité externe d'un cas unique ne vise pas la généralisation statistique, mais une généralisation analytique.

L'entreprise Mockup qui a été choisie correspond bien au type de cas recommandé par Stake. Elle a toutes les caractéristiques d'une entreprise de haute technologie, elle intervient dans un secteur très innovant, tels qu'ils ont été décrits précédemment. Elle a connu un processus de création particulièrement heurté et évolutif et, à ce titre, porteur d'enseignement. Le choix de cette entreprise est lié à la possibilité qui a été donnée au chercheur de disposer d'un accès privilégié à l'information. Observateur de la genèse du projet avant sa création de 1998 à 2000, il a ensuite été investisseur minoritaire et passif au cours de la période 2000-2004. L'étude de cas a donc été menée de manière longitudinale sur une période de six ans (1998-2004). L'observation s'est faite de manière ouverte, avec l'accord de l'entrepreneur. L'implication du chercheur en tant qu'investisseur pose la question du «paradoxe de l'intimité» (Mitchell, 1993). L'intimité a permis d'obtenir une information abondante, en continu et de première main. La distance a été rendue possible par la multiplicité des relations avec d'autres acteurs du projet tels que les employés et les investisseurs. Le temps et les difficultés rencontrés ont constamment amené le chercheur à analyser les 
actions menées et à développer une attitude critique. Au cours des années la prise de note a été continuelle, soit lors d'entretiens en face à face, soit lors d'entretiens téléphoniques.

L'analyse des données dans un cas longitudinal nécessite de prendre des précautions. À partir d'une matrice chronologique, l'analyse des données consistait à observer la création d'entreprise en portant attention à l'évolution des différents composants du système, puis à apprécier à partir de quelle intensité de changement on pouvait considérer qu'il y avait une évolution d'un projet à un autre.

\subsubsection{Présentation du cas}

L'entrepreneur qui a créé Mockup avait déjà été le créateur d'une société devenue le leader mondial des logiciels de conception assistée par ordinateur (CAO) pour architectes, dans les années 1980. À partir de 1996, habitant à Nice, il est à la recherche d'opportunités d'affaires dans le domaine de la 3D. Il identifie un modeleur ${ }^{2}$ 3D et s'associe avec son concepteur pour fonder un éditeur de logiciel. La société Mockup est créée en janvier 2000 avec l'aide de capital de proximité. Le siège est localisé à Montréal et l'équipe de développement à Nice. Afin de ne pas entrer en concurrence frontale avec les puissants éditeurs qui tiennent déjà le marché des modeleurs 3D, le produit est rapidement repositionné comme une visionneuse d'objets 3D. Les visionneuses existantes sont couplées aux modeleurs et permettent de visualiser les objets créés dans la continuité de l'environnement technique. La visionneuse de Mockup innove en permettant de rendre visible l'objet 3D par Internet, sur un ordinateur du marché et avec des fonctions de manipulation aisées et intuitives.

Le couple produit-marché retenu est donc une visionneuse 3D destinée aux architectes qui souhaitent réaliser des maquettes virtuelles pour leurs clients. La distribution du logiciel est prévue par téléchargement sur Internet. Une équipe chevronnée, disposant d'une expérience dans la 3D et le marché des architectes est constituée et répartie entre Montréal et Nice. Un financement de 1,7 million de dollars canadiens fournis par un fonds de capital-risque français en janvier 2001 permet le déploiement de l'entreprise. Une politique de partenariat avec des sites portails spécialisés et des actions de communication ciblées vers les architectes est mise en place. Le modèle économique est basé sur un coût faible du logiciel (100\$)

2. Un modeleur est une application logicielle permettant de concevoir des objets virtuels. Ils sont utilisés dans tous les secteurs de l'industrie pour la conception de produits simples ou complexes. 
dans le but de créer une base de clients. La vente ultérieure de versions plus élaborées doit générer des ventes récurrentes et de la marge. Malgré une mise en œuvre maîtrisée de la stratégie choisie, les résultats sont très décevants: quelques dizaines de ventes alors que plusieurs dizaines de milliers de téléchargement du logiciel de démonstration ont été faits. Ce mauvais résultat s'explique par la nouveauté de l'offre et l'absence de besoin des architectes.

Un changement stratégique majeur est alors engagé au cours de l'été 2001 avec un repositionnement du couple produit-marché. À partir de la même technologie, un nouveau produit est développé. Il est positionné comme un composant de visualisation 3D pour les applications logicielles de gestion du cycle de vie des usines ou des produits. Il permet aux équipes de conception de produits complexes ou d'usines d'échanger des images 3D des projets en cours de réalisation. Cette évolution du segment de marché des architectes vers celui des entreprises industrielles, ou d'ingénierie, induit des changements profonds dans les caractéristiques du projet. Le nouveau produit demande de très gros développements pour pouvoir être compatible avec les outils de CAO 3D du marché et s'intégrer dans les environnements informatiques des utilisateurs. Les ressources financières initiales épuisées, les effectifs doivent être réduits et l'équipe de direction est revue en fonction des nouvelles priorités. La stratégie commerciale évolue d'une distribution par Internet à la mise en place de partenariat avec des distributeurs et des contacts directs avec des clients de référence. Les moyens financiers nécessaires pour mener cette nouvelle stratégie sont estimés à 10 millions de dollars canadiens. En mars 2002, un investisseur de Montréal fournit deux millions de dollars canadiens. Le développement des nouveaux produits est plus long et plus coûteux que prévu. Une évolution de la force de vente est nécessaire compte tenu des caractéristiques des nouveaux clients. Du fait des retards du produit et des longs cycles de décisions des clients, les premières ventes ne se font qu'au début 2004. Entre-temps, les effectifs passent d'une vingtaine de personnes à moins de six personnes et la filiale française est mise en sommeil. Les capitaux levés au deuxième tour sont à nouveau consommés. Début 2004, le dirigeant de Mockup continue à mener des actions commerciales et des partenariats stratégiques avec très peu de moyens afin d'obtenir les preuves marché suffisantes pour une nouvelle collecte de fonds. La trésorerie est faite au coup par coup par les capitaux-risqueurs et le capital de proximité. 


\subsection{Un modèle composite pour restituer la complexité et l'évolutivité du processus de l'entrepreneuriat technologique}

Comme il a été rappelé dans la première partie, les modèles d'émergence des jeunes entreprises technologiques n'ont pas suffisamment pris en compte l'évolution et la complexité dans un contexte d'incertitude. Nous avons indiqué les emprunts qui ont été faits aux théories et modèles préexistants. À partir de ces éléments, la proposition faite considère que les phases par lesquelles passe la création sont en réalité des projets en eux-mêmes et que réussir une création d'entreprise, c'est réussir successivement trois projets, chaque projet étant considéré comme un système entrepreneurial.

\subsubsection{Piloter un système entrepreneurial}

Le processus de création d'une nouvelle entreprise consiste à piloter un système entrepreneurial. Les éléments du système sont l'entrepreneur, l'activité, l'organisation, l'environnement et les ressources financières qui ont été présentés et qui reprennent les propositions de Sammut. Certains ont été redéfinis, comme l'activité, qui est décrite par le triplet technologie-produitmarché, et l'environnement, qui a été décliné en trois sous-éléments: le milieu innovant, l'environnement personnel et l'environnement compétitif et réglementaire. La figure 1 représente le système entrepreneurial dont les éléments sont en interaction. En raison des particularités de la haute technologie, les changements dans les éléments sont fréquents et souvent de forte intensité, provoquant ainsi des interactions fortes entre les éléments, ce qui a pour effet de le rendre particulièrement instable.

\section{Figure 1}

\section{Le système entrepreneurial de la jeune entreprise technologique}

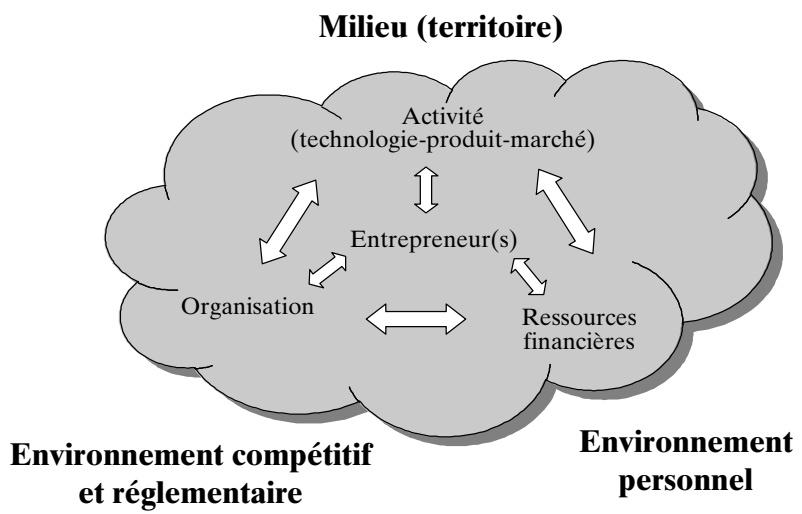

Revue internationale P.M.E., vol. 21, nº 1, 2008 
Le nuage comme forme de contenant a été préféré à une ellipse ou un rond qui délimiterait trop précisément le dedans et le dehors. Le nuage doit être interprété comme un contour flou et poreux. La représentation fait cependant le choix de situer les variables sur lesquelles les entrepreneurs ont un certain contrôle à l'intérieur du nuage. Les trois types d'environnements qui seront précisés ultérieurement, situés par convention à l'extérieur du nuage, sont individualisés pour souligner leur singularité. Les différents éléments vont être présentés et illustrés à partir du cas.

Le rôle de l'entrepreneur est central dans le processus de création d'entreprise. Dans les entreprises technologiques, de nombreuses études ont montré que l'entrepreneur est rarement seul, mais qu'il existe des équipes entrepreneuriales constituées de deux ou trois personnes. De plus, l'importance des équipes de fondateurs dans la construction de projets ambitieux à croissance rapide a été clairement mise en évidence (Cooper et Dailly, 1997; Cooney, 1999).

La relation étroite entre l'entrepreneur et l'entreprise en création a été qualifiée de dialogique (Bruyat, 1994), de dialectique (Verstraete, 2001) ou encore de situation entrepreneuriale par Fayolle (2004). Ces auteurs insistent sur l'évolution conjointe de l'entrepreneur et de son projet. Par conséquent, si l'on reconnaît l'existence d'équipes entrepreneuriales, il convient d'étendre la situation entrepreneuriale de l'individu à l'équipe. Cela signifie que l'on prend en compte son émergence et ses recompositions. Dans le cas de Mockup, l'équipe est initialement constituée autour du fondateur et de son associé développeur. L'historique du fondateur dans la CAO pour les architectes a largement conditionné le choix de l'activité et donc du projet. L'équipe va ensuite profondément évoluer au fur et à mesure des changements de l'activité et de l'état des ressources financières. Les entrées et les sorties des membres de l'équipe dirigeante auront finalement pour effet que le seul personnage pérenne sera le fondateur principal. Ces changements fréquents au sein de l'équipe auront de multiples effets sur les autres éléments du système.

Nous avons choisi de définir l'activité par le triplet technologie-produitmarché. L'activité de la jeune entreprise technologique est délicate à saisir car elle est évolutive et se construit chemin faisant. Millier (1999) a particulièrement bien montré que la définition de l'activité passe par des étapes de foisonnement, de segmentation et de focalisation et que l'atteinte de l'activité porteuse du développement de l'entreprise est un long processus.

L'activité initiale de Mockup peut être définie comme la fourniture d'une visionneuse d'objet 3D pour les architectes à partir d'une technologie propriétaire. Cette première activité a une incidence majeure sur les autres 
choix du système comme le recrutement d'un architecte et de collaborateurs de l'entreprise précédente ou encore la mise en place d'une commercialisation sur Internet. À la suite de l'échec de la première activité, la nouvelle activité devient un composant 3D de visualisation pour les applications logicielles de gestion du cycle de vie des usines ou des produits, à partir de la même technologie. Cette nouvelle activité bouleverse tout le système. Il s'avère de plus que la technologie n'est plus la même, puisque d'un produit autonome conçu pour les architectes, il faut désormais évoluer vers une solution compatible avec les outils du marché et s'intégrer dans les environnements informatiques des utilisateurs. Par rapport à la première activité, ce sont donc les trois composants, le produit, le marché et la technologie, qui ont été modifiés. Cette nouvelle activité nécessite de nouvelles ressources financières ainsi qu'une commerciale différente. Les besoins de développement techniques sont très importants et la localisation à Montréal devient moins judicieuse puisque les équipes de R-D sont en réalité restées en France.

Le rôle de l'organisation et son évolution ont été abondamment traités dans la littérature, en particulier en établissant les phases par lesquelles passent les entreprises. Dans le cas des jeunes entreprises technologiques, Blais et Toulouse (1992) ont constaté des degrés de formalisation différents des principales compétences de gestion en fonction des phases (intensité de la structuration, importance des systèmes de gestion et des procédures de planification). Julien (2001) met en évidence la nécessité de l'organisation de s'adapter par des pratiques d'autoréorganisation et une capacité à gérer l'improvisation. C'est ce que va faire Mockup. L'organisation va connaître des modifications majeures et parfois brutales. En 2001, l'équipe qui avait atteint 20 personnes revient à 7 personnes. Comme nous l'avons déjà indiqué, les évolutions dans les compétences sont importantes. Les besoins en expertise et les modes de fonctionnement ne sont pas en phase avec les changements de l'activité. Étant donné la double implantation, une double organisation se met en place sans une véritable répartition des tâches et un mode de coordination adapté. L'entrepreneur homme-orchestre s'épuise d'une localisation à l'autre sans régler ces dysfonctionnements.

L'environnement est omniprésent dans les approches de la création d'entreprise. L'environnement réglementaire et compétitif est bien pris en compte dans la démarche stratégique de la firme et ne mérite pas d'être décrit plus avant dans ce travail. Le modèle propose de rajouter l'environnement personnel et le milieu, c'est-à-dire le territoire dans lequel évolue l'entreprise.

L'environnement personnel de l'entrepreneur est un élément capital. L'entrepreneur, homme social, est un produit de son milieu (Fillion, 1997) et il dispose d'un capital social (Bourdieu 1981). C'est dans l'environnement 
personnel que les entrepreneurs vont puiser ressources, soutiens et crédibilité. L'environnement personnel du créateur de Mockup a joué un rôle important dans le projet. Il va aussi utiliser son capital social pour pourvoir le projet en ressources humaines. Nous l'avons déjà dit, plusieurs personnes de la première entreprise vont rejoindre Mockup, d'ailleurs peut-être plus pour des raisons de confiance que de compétences. Ce sera sans doute une force du projet, mais aussi une faiblesse. Par ailleurs, les parents du fondateur constituent des soutiens essentiels dans des moments difficiles, de même que quelques business angels qui ont établi des relations d'amitié avec l'entrepreneur.

À ces deux types d'environnements il faut ajouter le territoire ou, plus précisément, le milieu innovant tel qu'il a été proposé par Aydalot (1986) et le GREMI ${ }^{3}$. En effet, depuis Marshall (1920), sous des approches et des vocables différents, économistes, géographes et sociologues ont exploré les caractéristiques tangibles et intangibles des territoires et les avantages offerts aux entreprises. En effet, nous avons vu que le développement s'est fait sur une double localisation: Nice et Montréal. Justifié principalement pour des raisons fiscales de la R-D, ce choix a eu une incidence certaine sur les difficultés qu'a posées le projet. Outre les aspects organisationnels déjà cités, ces deux localisations ne vont pas permettre à l'entrepreneur et à l'entreprise de profiter pleinement des avantages des territoires. Ainsi, le siège étant à Montréal, le projet, considéré comme canadien, ne trouvera pas tous les soutiens du système d'innovation français et ceux du territoire de Sophia-Antipolis. Ainsi, l'ANVAR ne financera pas les développements technologiques du projet. C'est d'autant plus regrettable que la R-D qui aurait dû être à Montréal restera finalement à Nice pour des raisons de choix de mode de vie des personnes concernées! Ainsi, le projet n'aura finalement ni les effets bénéfiques de la fiscalité du Québec, ni ceux du soutien de l'ANVAR.

Le dernier élément du système entrepreneurial est constitué par les ressources financières. Celles-ci sont importantes du fait des spécificités des jeunes entreprises technologiques, d'une part, et du temps consacré par le dirigeant pour les mobiliser, d'autre part. Les spécificités sont le décalage entre les dépenses et les recettes, l'impact des décisions rapides sur la structure financière, l'importance des actifs immatériels et le besoin permanent d'évaluation de l'entreprise (Gasiglia, Gueye et Pistre, 2000). La multiplicité

3. GREMI, Groupe de recherche européen sur les milieux innovateurs. Les travaux GREMI 1 et GREMI 2 ont défini le concept de milieu et analysé les relations entre la firme innovante et le milieu. 
des acteurs du financement, leur spécialisation par phase et par secteur ainsi que la complexité des négociations expliquent le temps important consacré par le dirigeant.

Dans le cas de Mockup, on peut simplifier les observations en notant que l'entreprise a fait deux collectes de fonds. Mais ces collectes ont été longues et finalement les ressources financières n'ont pas été en phase avec les autres éléments du système. La première collecte de fonds a été entièrement consommée par une activité qui n'a pas trouvé son marché. La deuxième collecte de fonds sera consommée dans la transition entre les deux activités, mais n'a pas été mise à profit pour faire déboucher la nouvelle activité.

Appuyée sur les éléments du cas, la description du système entrepreneurial et de ses composantes permet d'identifier et d'analyser les évolutions de la jeune entreprise innovante. Mais cette représentation par un système n'est pas suffisante pour singulariser des moments critiques de la vie d'une jeune entreprise, ni pour mettre l'accent sur des modifications majeures du système. C'est pour cette raison que le système entrepreneurial est complété par la proposition des trois projets. En effet, comme indiqué précédemment, les évolutions majeures du système peuvent amener à une reconfiguration importante du projet d'entreprise. Ce n'est pas seulement le passage d'une phase à une autre, c'est le passage d'un projet à un autre. C'est la raison pour laquelle nous proposons de considérer que le système entrepreneurial passe par plusieurs projets différents.

\subsubsection{Réussir une création d'entreprise, c’est réussir trois projets}

En s'inspirant des trois phases de Bygrave et Hofer (1991) et de leurs variantes, nous proposons de considérer qu'une jeune entreprise en création passe nécessairement par trois projets précis : le projet d'affaires, le projet d'entreprendre et le projet d'entreprise. Le passage des trois phases au trois projets n'est pas une tentative de réappropriation de l'existant. C'est une avancée qui permet de rendre plus explicites les enjeux de chacune des étapes et qui met l'accent sur l'éventuelle absence de continuité naturelle entre elles. Les termes projet d'affaires, projet d'entreprendre et projet d'entreprise peuvent prêter à confusion avec des usages existants. En particulier, le terme projet d'entreprise ici ne fait pas référence à l'existence juridique du projet, mais à un état du système adapté au développement de l'activité économique.

À l'origine d'une démarche entrepreneuriale, il y a l'identification d'une opportunité d'affaires par des individus qui deviennent potentiellement des entrepreneurs. À l'issue d'un processus de création d'une occasion d'affaires, ils structurent une proposition que nous appellerons projet d'affaires. 
À ce stade, ils disposent fréquemment d'un plan d'affaires, mais pas nécessairement. Le système entrepreneurial comprend les éléments, mais ils sont incomplets et les interactions entre eux sont faibles et souvent mal identifiées.

S'ils décident d'aller de l'avant, ces entrepreneurs vont s'engager dans le projet d'entreprendre pour tenter de transformer l'opportunité d'affaires en activité économique. Le système entrepreneurial se renforce. Les éléments sont mieux mobilisés et les interactions se mettent progressivement en place et se complexifient. Le projet d'entreprendre s'adapte et se transforme pour tenir compte des faits et des apprentissages. Si le modèle économique n'est pas trouvé ou la gestion du système déficiente, le projet sera vendu ou disparaîtra et n'atteindra jamais le projet d'entreprise. Si ces évolutions sont favorables, l'opportunité d'affaires devient une activité économique validée par l'atteinte d'un modèle économique prouvé.

Les entrepreneurs peuvent alors passer au projet d'entreprise qui a pour objet de bâtir l'entreprise qui convient à l'activité réelle de l'entreprise. Comme on sait que les premières années sont souvent l'objet de tâtonnements, voire de répétition, le projet d'entreprise sera proche ou significativement distinct du projet d'entreprendre dont il est issu. Les principaux risques ont été éliminés, les orientations sont mieux affirmées et les routines professionnelles et organisationnelles commencent à être maîtrisées. Dans ce projet, les interrelations des éléments du système entrepreneurial sont mobilisées et coordonnées en fonction de l'activité. Une fois le projet d'entreprise maîtrisé, son analyse rentre dans des cadres balisés par la stratégie d'entreprise et la littérature sur les organisations.

Nous pensons que les trois projets présentés dans ce travail sont identifiables dans toutes les créations d'entreprises technologiques. Ce qui va considérablement varier d'une entreprise à l'autre, c'est le niveau de similarité entre les trois projets et les modalités de passage de l'un à l'autre. On peut représenter la création comme un projet par une juxtaposition de nuages (figure 2), chaque nuage étant lui-même un système entrepreneurial. La position relative des nuages constitue une appréciation de la proximité entre les différents projets. Cette représentation limite la détermination séquentielle des modèles processuels et permet de prendre en compte des situations très différentes dans lesquelles les écarts entre les projets peuvent être importants ou plus limités, comme le montrent les différentes configurations théoriques de la figure 2 . 


\section{FIGURE 2}

\section{Deux configurations possibles des trois projets}

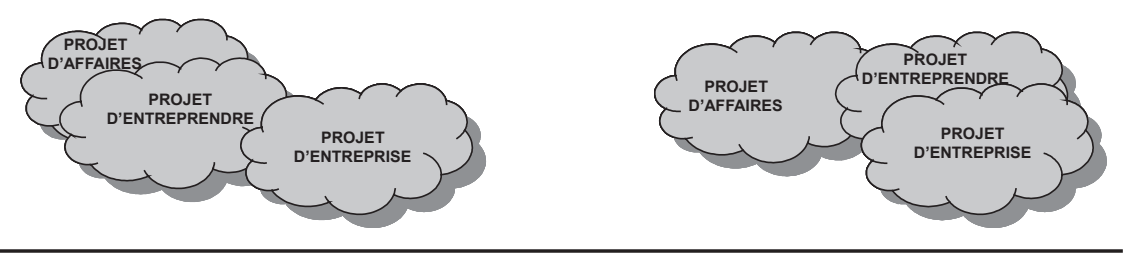

Dans le cas de Mockup, le projet d'affaires a été long à se dessiner. L'entrepreneur n'était pas à l'origine de la technologie, mais le projet d'affaires a bénéficié de son expérience préalable dans ce domaine de la conception assistée par ordinateur. Cette expérience préalable a constitué un atout dans la structuration de l'opportunité d'affaires et dans la capacité à bâtir un plan d'affaires convaincant. On peut considérer que l'entrepreneur a mobilisé progressivement et efficacement les éléments du système dans cette phase.

On observe également que le projet d'entreprendre a été engagé presque en même temps que le projet d'affaires puisque la décision d'entreprendre était prise avant même la phase d'analyse et qu'une partie des éléments du projet d'entreprendre avaient déjà été mis en œuvre, comme, par exemple, le prérecrutement de l'équipe, formée principalement de collaborateurs de la première entreprise. Notons que cet engagement simultané des deux projets est fréquent. Il ne préjuge en rien de la réussite ou de l'échec du projet, mais il peut entraîner une précipitation en négligeant l'analyse de l'opportunité. La fréquence de l'existence simultanée du double projet rend d'autant plus nécessaire l'identification de leurs caractéristiques respectives. Dans le cas de Mockup, la confusion des deux projets peut être considérée comme une erreur, trop d'éléments clés du système ayant été mis en place avant une validation de la cohérence du projet d'affaires. L'expérience préalable l'a emporté sur l'analyse et les moyens mobilisés n'ont pas été suffisamment adaptés à la situation. Le développement simultané ou légèrement différé des deux projets est aisément pris en compte par le modèle, soit analytiquement, comme cela vient d'être fait, soit par la représentation graphique ci-contre (figure 3). 
Figure 3

Le cas Mockup représenté selon le modèle des trois projets

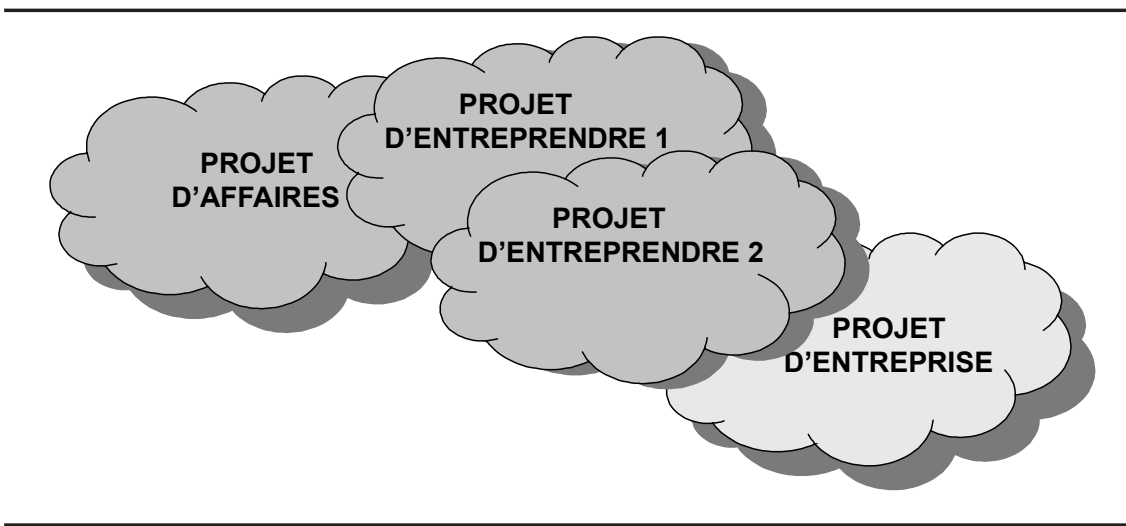

L'échec de l'offre initiale pour les architectes n'a pas permis de transformer le projet d'entreprendre en projet d'entreprise. Il a été nécessaire de redéfinir une nouvelle activité, c'est-à-dire une nouvelle combinatoire technologie-produit-marché en repositionnant le produit comme un composant 3D de visualisation pour les applications logicielles du cycle de vie des usines ou des produits. C'est en réalité un deuxième projet d'entreprendre qui a été redéfini, du fait de changements importants ou très importants dans les éléments. Le passage du premier au second projet d'entreprendre se fait dans une certaine discontinuité qui remet fortement en cause les interactions entre les éléments qui préexistaient. Cette situation illustre bien les allers et retours et les boucles rétroactives signalés par la littérature. Encore une fois, le modèle restitue aisément cette situation qui est représentée par deux nuages distanciés. Courant 2004, le projet d'entreprise reste toujours en devenir et dépendra de la confirmation des premières ventes en cours.

Le cas Mockup peut être représenté par le modèle des trois projets. Le processus de création de Mockup est décrit tout d'abord comme une relative confusion du projet d'affaires et du projet d'entreprendre, puis par la réalisation d'un deuxième projet d'entreprendre. L'intérêt d'avoir considéré le processus de création comme trois projets et non pas comme un seul projet évolutif apparaît justifié : il montre en effet que chaque projet est en enjeu en soi, que le passage de l'un à l'autre n'a rien de naturel et qu'il n'a rien de linéaire. 


\section{Conclusion}

L'objectif de ce travail était de proposer aux entrepreneurs et aux acteurs de l'accompagnement une représentation de la création d'entreprise technologique qui permette de mieux prendre en compte un phénomène complexe, particulièrement évolutif et non linéaire, trois caractéristiques inhérentes à l'innovation technologique.

Le modèle permet de bien prendre en compte la particularité du cheminement du projet sans chercher à l'enfermer dans un cadre prédéfini. $\mathrm{Au}$ contraire, il peut s'adapter à une situation particulière. Même parfois concomitants ou répétés, les trois projets apparaissent comme pertinents pour décrire la situation. Le choix des trois projets et des cinq éléments du système entrepreneurial peut naturellement être discuté. Les trois projets sont largement inspirés des étapes du processus entrepreneurial proposées par de nombreux auteurs et les cinq éléments sont également directement extraits de la littérature. L'originalité de la démarche tient donc à la construction d'un modèle composite qui emprunte à différentes approches. Les apports de l'approche processuelle tiennent aux phases et aux interactions et ceux de l'approche systémique, à la conception même du modèle. L'approche par le projet vient donner son sens à l'ensemble, en particulier en identifiant trois projets distincts au lieu d'un seul évolutif.

Le modèle favorise l'apprentissage des entrepreneurs. Proposer trois projets rompt avec la représentation commune d'une évolution basée sur le passage plus ou moins linéaire d'un état à un autre. Définir chaque projet comme un système permet en outre d'engager des réflexions et analyses sur les éléments, leurs interactions et le sens de la dynamique. Ces réflexions constituent une base de départ d'une acquisition de connaissance qui ne se fait pas de manière fonctionnelle mais de manière transactionnelle, les composants du système de gestion n'étant pas seulement abordés pour eux-mêmes mais en relation dynamique avec les autres. Cette représentation présente également la vertu de faciliter la communication entre les entrepreneurs et les accompagnateurs. En effet, son caractère stratégique, projectif et évolutif permet d'engager un dialogue sur l'essentiel: identifier conjointement l'état dans lequel est le projet à un moment donné et anticiper ses évolutions.

Un modèle est une représentation simplifiée d'un processus ou d'un système, destinée à expliquer ou à simuler une situation réelle. Le modèle des trois projets ne propose pas d'établir de relations causales entre les variables du système. Il doit plutôt être considéré comme un modèle de type descriptif, voire de simulation. Il permet à la fois d'analyser une situation ex-post, et de simuler ex-ante des scénarios d'évolution de la création d'entreprise. Dans 
cette fonction de simulation, on peut en effet, à partir de l'évolution d'une des variables du système, réfléchir sur l'évolution des autres variables et sur le projet dans son ensemble.

On peut s'interroger sur la pertinence d'un modèle qui emprunte à plusieurs approches. Comme Koenig (1993), nous pensons qu'au contraire leur pluralité est une opportunité d'enrichissement de la connaissance. Il faut d'ailleurs noter à ce propos que les différentes démarches présentées séparément ne le sont pas nécessairement et qu'en réalité, avec le temps, les approches les plus récentes se sont déjà enrichies des précédentes.

Si ce modèle semble utile par rapport à son objectif, on peut s'interroger sur sa validité. Dans le cadre de la démarche constructiviste, l'approche est à finalité interprétative et la nature de la connaissance produite est subjective et contextuelle. La validité doit être jugée par rapport à l'intention. Ce modèle n'est bien entendu pas exempt de limites. Des travaux complémentaires devront porter sur la pertinence du découpage en trois projets et sur le passage d'un projet à l'autre. Enfin, la dynamique des éléments de chaque projet devrait être mieux analysée et conceptualisée.

Le modèle proposé devrait trouver un usage dans la formation des entrepreneurs. À ce titre, il répondrait en partie aux suggestions de Massacrier et Rigaud qui appelaient de leurs vœux des représentations permettant aux entrepreneurs de prendre conscience des sources probables d'écart entre leurs premières estimations et les réalisations effectives, en les aidant à tester eux-mêmes leurs projets.

\section{Bibliographie}

Albert, P. et P. Mougenot (1998), «La création d'entreprises high-tech», Revue française de gestion, mars-avril-mai, vol. 24, p. 106-119.

Albert, P., A. Fayolle et S. Marion (1994), «L'évolution des systèmes d'appui à la création d'entreprise », Revue française de gestion, novembre-décembre, vol. 20, $\mathrm{n}^{\circ} 101, \mathrm{p} .100-112$.

Aydalot, P. (dir.) (1986), Milieux innovateurs en Europe, Paris, GREMI, C3E.

BARUCH, Y.(1997), «High technology organization: what it is, what it isn’t», International Journal of Technology Management, vol. 13, n 2, p. 179-199.

Bernasconi, M. et F. Moreau (2003), «L'évolution du projet des jeunes entreprises technologiques innovantes au cours des premières années: une méthode d'appréciation du cheminement stratégique», Revue internationale PME, vol. $16, \mathrm{n}^{\text {os }} 3-4$, p. 11-29.

Revue internationale P.M.E., vol. 21, nº 1, 2008 
Blais, R. et J.M. Toulouse (1992), Entrepreneurship technologique: 21 cas de PME à succès, Montréal, Éditions Transcontinentales/Éditions de la Fondation de l'Entrepreneurship.

Bourdieu, P. (1981), «Le capital social: notes provisoires», Actes de la Recherche en Sciences sociales, vol. 3, p. 2-3.

BRÉCHET, J.-P. (1994), «Du projet d'entreprendre au projet d'entreprise», Revue française de gestion, $\mathrm{n}^{\circ}$ 99, p. 5-13.

BRUYAT, C. (1994), «Contributions épistémologiques au domaine de l'entrepreneuriat», Revue française de gestion, vol. 20, $\mathrm{n}^{\circ}$ 101, p. 87-99.

Bygrave, W.D. et C.W. Hofer (1991), «Theorizing about entrepreneurship», Entrepreneurship Theory \& Practice, vol. 16, n 2 , p. 13-22.

Churchill, N. (1998), «Les six phases de la croissance», L'art d'entreprendre, Paris, Les échos-Village Mondial.

Churchill, N. et V. Lewis (1983), «The five stages of small business growth», Harvard Business Review, vol. 61, p. 30-50.

Cooney, T.M. (1999), «What is the relationship between entrepreneurial teams, structure and strategy in fast growing firms? », ICSB 44th World Conference, Naples, Juin.

Cooper, A.C. (1986), «Entrepreneurship and high technology», dans D.L. Sexton et R.W. Smilor (dir.), The Art and Science of Entrepreneurship, Cambridge, Mass., Ballinger.

Cooper, A.C. et C.M. DAilly (1997), «Entrepreneurial teams», dans D.L. Sexton et R.W. Smilor (dir.), Entrepreneurship 2000, Chicago, Illinois, Upstart Publishing, p. $167-188$.

De FilipPi, R. et M. Arthur (1998), «Paradox in project-based enterprise: the case of film making», California Management Review, vol. 40, nº 1, p. 186-91.

Eggers, J., K. Leahy et N. Churchill (1994), «Stages of small business growth revisited: insight into growth path and leadership/management skills in low-and high growth companies », Cahier de recherche, INSEAD, 94/63/ENT.

FAyolle, A. (2004), Entrepreneuriat, Paris, Dunod.

Fillion, L.J.(1997), «Le champs de l'entrepreneuriat: histoire, évolutions, tendances », Cahier de recherche n ${ }^{\circ} 97.01$, HEC Montréal.

GADDIs, P.O. (1959), «The project manager», Harvard Business Review, mai-juin, p. 89-97.

GARNSEY,E. (1996), «A new theory of the early growth of the firm», 41st ISCB World Conference, Stockholm, juin.

GARTNER, W.B. (1985), «A framework for describing the phenomenon of new venture creation », Academy of Management Review, vol. 10, p. 696-706.

Gasiglia, C., S. Gueye et N. Pistre (2000), «La valorisation, enjeu central de la logique financière des start-up», dans M. Bernasconi et M. Monsted (dir.), Start-up High-Tech, Paris, Dunod, p. 167-177.

Revue internationale P.M.E., vol. 21, nº 1, 2008 
Gordon Bell, C. et J. McNamara (1991), High-Tech Ventures, Reading, MA, AddisonWesley.

GREINER, L. (1972-1998), «Evolution and revolution as organizations grow», Harvard Business Review, juillet-août 1972, mai-juin 1998 (2éd.).

Hernandez, E.M. (2001), L'entrepreneuriat: Approche théorique, Paris, L'Harmattan.

JuLien, P.-A. (2001), «Les PME à forte croissance et la métaphore du jazz. Comment gérer l'improvisation de façon cohérente», Revue internationale PME, vol.14, $\mathrm{n}^{\text {os }} 3-4$.

KLofsten, M. (1997), «Management of the early development process in technology based firm », dans D. Jones-Evans et M. Klofsten (dir.), Technology, Innovation and Enterprise, Londres, Macmillan Press.

KNights, D. et F. Mueller (2004), «Strategy as a project: overcoming dualisms in strategy debate», European Management Review, vol. 1, p. 55-61.

Koenig, G. (1993), «Production de la connaissance et constitution des pratiques organisationnelles », Revue de gestion des ressources humaines, $\mathrm{n}^{\circ}$ 9, novembre, p. 4-17.

Le Moigne, J.L. (1977), Théorie du système général, Théorie de la modélisation, Paris, Presses universitaires de France, Réédition 1994.

Marchesnay, M. (2002), «Pour une approche entrepreneuriale de la dynamique ressources-compétences. Essai de praxéologie», Éditions de l'ADREG, <www. editions-adreg.net $>$.

Marshall, A. (1920), Principles of Economics, Londres, Macmillan Press.

Massacrier, G. et G. Rigaud (1984), «Le démarrage d'activités nouvelles: aléas et processus », Revue française de gestion, mars-avril-mai, p. 5-18.

Millier, P. (1999), Marketing the Unknown:Developing Market Strategies for Technical Innovations, Chichester, New York, Wiley.

Mitchell, R.G. (1993), Secrecy and Fieldworks, Newbury Park, Cal., Sage Publications.

Monsted, M. (2000), «L'incertitude, source de risque et d'opportunités en high-tech», dans M. Bernasconi et M. Monsted (dir.), Start-up High-Tech, Paris, Dunod, p. $15-24$.

Neisheim, J. (2000), High Tech Start-up, Saratoga, Cal., Neishem.

QuinN, R. et K. CAMERon (1983), «Organizational life cycles and shifting criteria of effectiveness : some preliminary evidence», Management Science, vol. 29, $\mathrm{n}^{\circ} 1$, p. 33-51.

Sammut, S. (2001), «Processus de démarrage en petite entreprise: système de gestion et scénarios », Revue de l'Entrepreneuriat, vol. 1, n 1, p. 61-76.

SAPORTA, B. (1994), «La création d'entreprise: enjeux et perspectives», Revue française de gestion, vol. 101, $\mathrm{n}^{\text {os }} 11-12$, p. 74-86.

Revue internationale P.M.E., vol. 21, nº 1, 2008 
Shane, S. et S. Venkataraman (2000), «The promise of entrepreneurship as a field of research », Academy of Management Review, vol. 25, n 1, p. 217-226.

SöDERLund, J. (2004), «Building theories of project management: past research, questions for the future », International Journal of Project Management, vol. 22, p. 183-191.

Stake, R.E. (1994), «Case studies», dans N.K. Denzin et Y.S. Lincoln (dir), Strategies of Qualitative Inquiry, vol. 2, Newbury Park, Cal., Sage Publications, chapitre 4, p. 86-109.

Tesfaye, B. (1997), «Patterns of formation and development of high-technology entrepreneurs », dans D. Jones-Evans et M. Klofsten (dir.), Technology, Innovation and Enterprise, Londres, Macmillan Press, p. 61-106.

Twaalfhoven, B.M. et V. Kugi (2003), «Red paper on entrepreneurship, follow up to green paper entrepreneurship in Europe», European Ideas Network, Job Creation Seminar, Bruxelles, 10 juin.

Venkataraman, S. (1997), «The distinctive domain of entrepreneurship research», dans J.A. Katz (dir.), Advances in Entrepreneurship, Firm, Emergence and Growth., vol. 3, Greenwich, CT, JAI Press, p. 119-138.

Verstraete, T. (2001), «Entrepreneuriat: modélisation du phénomène», Revue de l'Entrepreneuriat, vol. 1, $\mathrm{n}^{\circ}$ 1, p. 5-23.

Yin, R.K. (1989), Case Study Research: Design and Method, Second Edition, Applied Social Research Methods Series, vol. 5, Newbury Park, Cal., Sage Publications. 